$16^{\text {th }}$ International Congress of Metrology, 06003 (2013)

DOI: $10.1051 /$ metrology/201306003

(C) Owned by the authors, published by EDP Sciences, 2013

\title{
A new Concept for High-Speed atline and inlineCT for up to $100 \%$ Mass Production Process Control
}

\author{
Oliver BRUNKE ${ }^{1}$, Jens LÜBBEHÜSEN ${ }^{1}$, Ferdinand HANSEN ${ }^{2}$ and Franz Friedrich BUTZ \\ GE Sensing \& Inspection Technologies GmbH, Product Management for 3D Metrology and Failure Analysis using \\ Computer Tomography, 31515 Wunstorf, Germany; Phone: +49 5031172 142; e-mail: oliver.brunke@ge.com \\ Volkswagen Foundry, Process Development, Hannover, Germany \\ ${ }^{4}$ Volkswagen Foundry, Head of Foundry, Hannover, Germany
}

\begin{abstract}
In manufacturing processes, inspection capability is increasingly used as early in the production value chain as possible to ensure that scrap and rework costs are minimised. Fast radiographic in-line inspection systems, fully unmanned and equipped with automatic defect recognition e.g. of castings, are now state-of-the-art technology. This paper explains the next evolutionary step within the X-ray technology of utilizing fast computer tomography technology, scanning concepts and algorithms fulfilling high- throughput requirements for different applications and first results of an atline / inlineCT evaluation at Volkswagen Foundry Hannover, Germany.
\end{abstract}

Keywords: Inline CT, Atline CT, Gantry based CT, Helix CT, Fast CT, 3D ADR, Automatic Defect Detection

\section{Introduction}

The automotive casting industry's typical throughput requirements ranges from 10 second for smaller piston or suspension parts up to 80-90 second for a complex engine part e.g. cylinder head. To meet this throughput requirement, a fully automated inspection method including the entire acquisition and analysis process is therefore vital. Main factors driving the cycle time of a fast inlineCT system are technologies used for efficient part handling, data acquisition, reconstruction and analysis. Using volumetric data and three-dimensional analysis has several advantages over the conventional radiographic 2D inspection: Depending on the sample size and X-ray penetration length, it allows reducing the scrap rate by describing the first time the 3D position and size of defects inside the volume while taking further machining steps into account. Detected anomalies can be analyzed in relation to final, machined areas and surfaces. Flaws within areas of a part that will be machined in a further processing step can be identified and disregarded as defects. In addition it can determine if porosities will open up to the surface. Actual part feature can be compared to nominal CAD data tolerances. With this ability, any variation in shape or size can be identified in a very early stage of the production process. And finally, foreign materials like inclusions or sand core remains may be detected, located and classified according to its density and position depending on their size and absorption behaviour. In all of these cases an immediate response to manufacturing process parameters can lead to high productivity.

The presented $\mathrm{CT}$ inspection system generation allows typical scan and inspection speed of 5 to 10 or more millimetres per second and thereby offering up to 200x higher throughput compared to typical industrial fan beam CT systems. In order to ensure the required image quality at short acquisition times it is equipped with a high power tube and efficient multiline detector, which leads to the right compromise between the amount of scattered radiation and scan time especially when attempting to inspect parts of larger dimensions. The part-handling concept supports a mixture of different part types and is designed for integration into production lines. 
Non-destructive inspection methods are used as early as possible in the value-added chain during industrial production processes to minimise costs and reworking. High-speed radiographic 2D inline inspection systems with automatic defect analysis software are state-of-the-art for the inspection of castings especially in the automotive industry. This paper describes the next stage of evolution within Xray technology, namely high-speed CT technology based on highly developed medical tomography systems which make it possible to carry out one hundred percent 3D inspections for a very wide range of industrial applications even at high throughput rates. High-speed CT is the only method currently available which allows internal structures of extremely complex components made of cast alloys, such as wall thicknesses in cylinder heads, to be inspected on a non-destructive basis directly in or close to the production line.

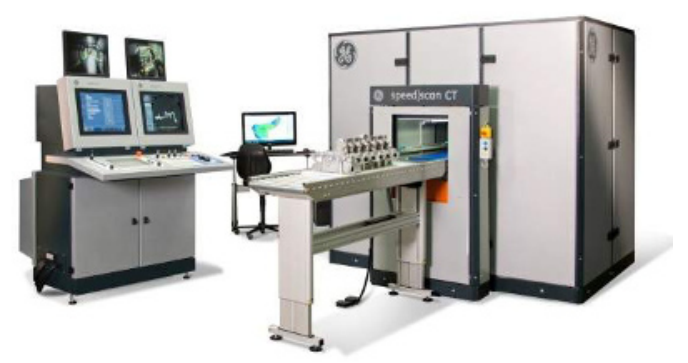

FIGURE 1: GE's fast CT system speed|scan using modified medical scanners allows components to be transported continuously at high speed through the rotating CT gantry of the tomograph, where they undergo a scan. The threedimensional evaluation is performed at both workstations.

Industrial process monitoring using volumetric data and three-dimensional analysis offers several advantages compared with conventional radioscopic $2 \mathrm{D}$ inspection. It allows the reject rate to be reduced by analysing the 3D position, form and size of the defects taking into account the subsequent processes the products must undergo. This means that any identified anomalies can be analysed with a view to the final, finished areas and surface finishes. Defects (flaws) in workpiece sections which will be removed by subsequent stages of the process, such as machining, can be ignored. An example illustrating these possibilities is shown in figure 2 .

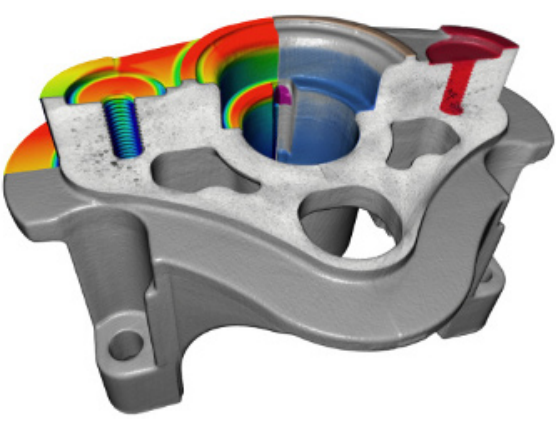

FIGURE 2: Comparison between $\mathrm{CT}$ and $\mathrm{CAD}$ data showing non critical defects in a region to be removed later.

Moreover, to avoid expensive scrap it is possible to check whether identified porous areas will be open on the final surface before processing begins. At the same time, the scanned workpiece geometry can be checked for anomalies using the nominal CAD data. This means that any form and size deviations e.g. due to wearing out of the casting mould can be identified at an early stage of the production process. And finally, foreign substances such as inclusions or residual sand casting core deposits may be identified, localised and classified in terms of their density and position. In all these cases productivity can be increased, since the process parameters can be immediately adjusted to counter any identified defects/flaws.

\section{Established medical technology for industrial process control}

Whilst computer tomography has been used in medicine for decades, over the last few years industrial CT has developed into a widely used 3D inspection method for scientific and industrial applications. In the latter case, the sample rotates in the X-ray beam which, thanks to its flexible enlargement principle, allows extremely high resolutions of just a few micrometres or even better to be achieved. However, in medical tomography, the CT gantry rotates with its generator, X-ray tube and corresponding detector at high speed around a patient lying on an imaging table which moves through the scanner. Although the fixed magnification limits the local resolution to typically a few hundred micrometres, the benefits of this technology become significant when this resolution is completely adequate, as is the case for analysing flaws in medium 
to large size light-metal castings. Whilst in the past, industrial inline CT applications have focussed on automatic loading and unloading devices using robot arms, for example, and on flat panel detectors with comparatively long acquisition times and only a rather small imageable area, the use of a gantry scanner means that the workpieces can be transported simply and continuously through the tomograph on a conveyor belt, with simple handling, and scanned comparatively quickly using helix multi-line technology. In addition, the throughput principle also means that various types of components can be inspected in sequence.

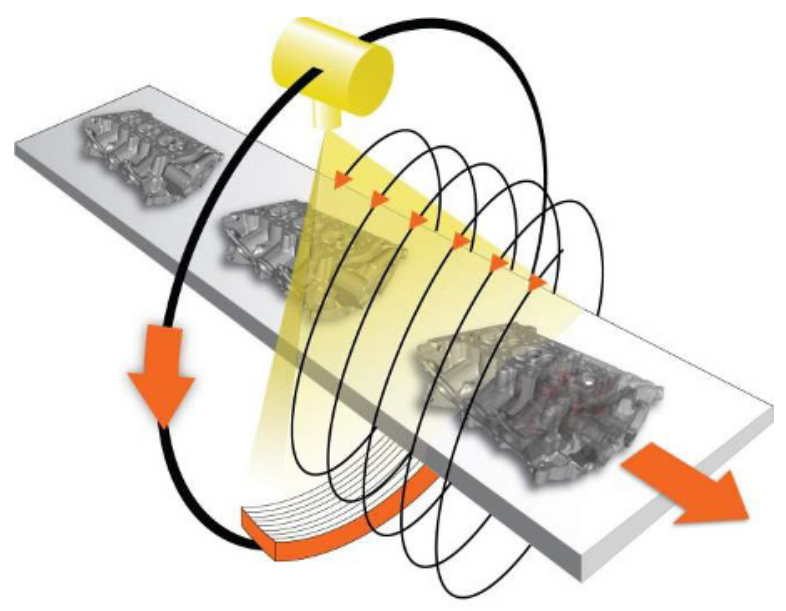

FIGURE 3: With the high-speed automatic helix CT, a gantry with an X-ray tube and corresponding multi-line detector rotates around the workpieces on a conveyor belt

\section{High-performance CT scanner for improved sample throughput}

The concept for the fast industrial inline CT system is based on medical tomography systems from GE Healthcare which have been adapted by GE Inspection Technologies with the appropriate, accurate transport facilities and automated 3D failure evaluation software modules for continuous operation in high-speed industrial inspection systems. A specially developed air-conditioned safety cabinet not only protects the surrounding area from the X-ray radiation, but also protects the tomograph from the dust and heat generated in harsh production environments.

The typical throughput requirements for the automotive foundry industry extend from 10 seconds for small pistons or chassis components to as much as 80-90 seconds for complex engine components such as cylinder heads. A fully automatic inspection method, including the whole data acquisition and analysis process, is required to meet these cycle times. The GE inlineCT scanner enables a typical scanning and inspection speed of 5 to 10 millimetres crosssection per second or more - a very high throughput rate compared to typical industrial CT systems. To guarantee the required image quality with short measuring times, the system is fitted with a high performance X-ray tube and a flexible 16-line detector which represents an efficient compromise between imaging artifacts caused by scattered radiation and scanning time - particularly for large components. Although the scanning time could be reduced considerably further by the use of detectors with 64 or more lines, the proportion of scattered radiation rises as the number of image lines increases and results in a major reduction in image quality and, therefore, less precise inspection results. Although this is tolerable for scanning organic bodies, light metal components in particular generate considerably higher scattered radiation.

The required X-ray radiation is generated by a specially-cooled GE rotary anode X-ray tube which ensures the high speed and complete irradiation of the component with a tube voltage of up to $140 \mathrm{kV}$ and a tube power of several $\mathrm{kW}$. The tube operating parameters have been adapted to meet the requirements of three-shift production in a 24/7 operation. Despite their high power these tubes function with a relatively small focus and therefore produce excellent image clarity. The data acquisition procedure uses a helix scan to ensure that the components for inspection can be fed through the system quickly and continuously. In this case, the tomograph rotates around the component. At relatively slow conveyor speeds, the various turns of the helix scan will overlap, which enables extremely high image quality to be achieved, but also results in a lower throughput speed. By carefully selecting the helix gradient, it is possible to achieve an appropriate compromise between scanning speed and resultant quality for the application in hand. A conveyor speed of up to $>30 \mathrm{~mm} / \mathrm{s}$ can be achieved for high speed scans. 


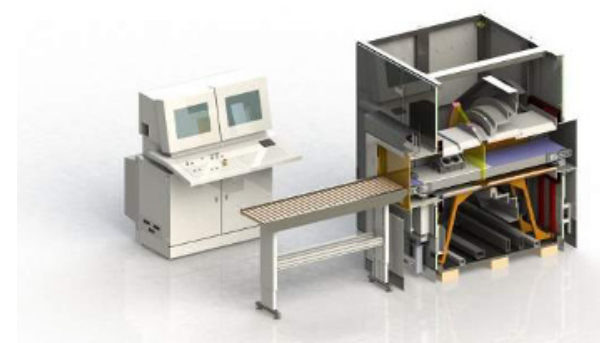

FIGURE 4: The GE speed|scan CT scanner is fitted with automatic conveyor equipment and encased in a radiation protection cabinet for industrial use.

\section{Integration of the CT scanner in the production line}

Different types of loading are possible depending on the area of application. In the first version of GE's new speed|scan system, the workpieces are moved off the production line by hand and placed on to a conveyor belt passing through the tomograph, which is hermetically encased with lead for radiation protection purposes and then moved out of it after the scan. (Fig. 4) This method has the benefit that random samples of up to $800 \mathrm{~mm} \times 400 \mathrm{~mm} \times 300 \mathrm{~mm}$ $(\mathrm{LxWxH})$ from various production lines can be scanned in parallel and at high speed. In contrast to continuous operation, an even higher rating of up to $53 \mathrm{~kW}$ is possible for this version.

The parts-handling system supports a mixture of all types of components and is also designed for direct integration into a production line. In this configuration, the components for inspection are transported into the tomograph on conveyor belts or pallet transport systems. Automatic gates ensure that no radiation can escape during the loading and unloading process, thus ensuring a safe and continuous inspection process.

FIGURE 5: The 3D defect detection and evaluation

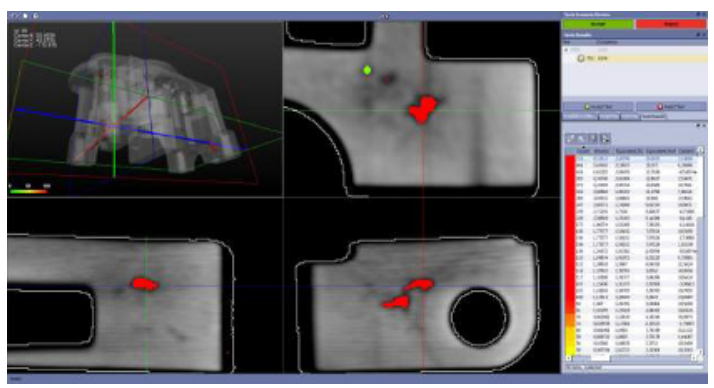

analysis is automatically performed parallel to the scan and reconstruction process.

\section{Evaluation parallel to the scanning process}

In addition to efficient sample handling and data recording, the main factors which decide the cycle time of a high-speed inline CT system include a parallel, fully-automatic 3D reconstruction and analysis process. This includes an automatic beam hardening correction procedure. The evaluation processes programmed for the particular workpiece are carried out automatically on the reconstructed 3D volume parallel to the scanning process. Typically achievable evaluation values are shown in tab. 1. For metrological applications, for example, the workpiece surface including all the undercuts are extracted; the 3D measurements are then carried out using preprogrammed measuring routines by special programs such as Polyworks Inspector ${ }^{\circledR}$ (InnovMetric Software Inc., Canada). Automatic porosity analyses in castings can be carried out using the new 3D automatic defect recognition (ADR) Software from GE on the basis of the full 3D volumetric dataset.

\begin{tabular}{|l|l|}
\hline & speed|scan fast atline/ inlineCT \\
\hline $\begin{array}{l}\text { Detail } \\
\text { detectability }\end{array}$ & $>325 \mu \mathrm{m}$ \\
\hline 3D metrology & $\begin{array}{l}\text { Sigma up to }<2 \mu \mathrm{m}, \text { absolute } \\
\text { deviation up to }<10 \mu \mathrm{m} \text { (based } \\
\text { on measurement on a standard } \\
\text { test phantom).This means that } \\
\text { tolerances of up to } 200 \mu \mathrm{m} \text { can be } \\
\text { measured. }\end{array}$ \\
\hline Defect detection & $\begin{array}{l}\text { Shrinkage cavities and pores } \\
\text { down to }>0.5 \mathrm{~mm}\end{array}$ \\
\hline
\end{tabular}

TABLE 1: speed|scan: Typically achievable values

\section{Metrological Application}

A comparison between reconstructed $3 \mathrm{D}$ data volume of fast GE CT and 3D data volume of a classical 2D fan beam CT system was carried out with focus to inner wall thickness of a cylinder head (Figure 6).

The measured value of wall thickness is in a region with normal penetration length through Aluminium $(<$ $200 \mathrm{~mm}$ ) nearly the same as measured in data volume of classical 2D CT scan.

Details: fast GE CT $4.37 \mathrm{~mm}$ against $4.38 \mathrm{~mm}$ of classical 2D CT, the actual wall thickness is $4.35 \mathrm{~mm}$. The used scan time of fast GE CT was a few hundred times faster than scan time of classical CT. In regions 
with high penetration length through Aluminium measurements deviate at present more from real wall thickness, but this will be compensated by software correction methods.

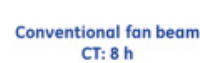

D

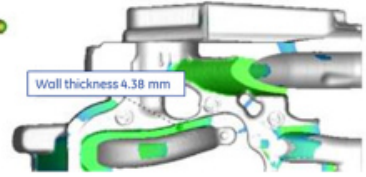

GE speed|scan CT: 1 mir

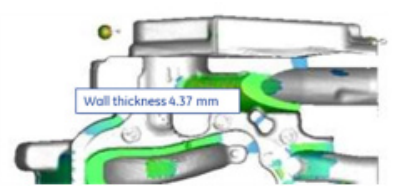

FIGURE 6: 3D wall thickness analysis on a cylinder head volume data set.

\section{Conclusion: New methods in industrial process monitoring using \\ CT}

GE's advanced medical gantry computer tomography provides technology that has been established and developed over a period of three-and-a-half decades. With GE's new industrial CT concept, it is now going to be also available for high speed and up to $100 \%$ inprocess inspections within the typical cycle times used in production lines such as those used in foundries. Simply transporting the components through the tomograph and scanning them at speeds of up to several centimetres per second after which they can be automatically evaluated. This opens up a whole host of new applications for in-line process control, which have in the past been out of reach of conventional industrial CT. 